\title{
Low energy dissipation readout of single-molecule ferroelectronic states by a spin-Seebeck signal
}

\author{
Hua-Hua Fu, ${ }^{1, *}$ Dan-Dan Wu, ${ }^{2}$ Gui-Fang Du, ${ }^{1}$ Qing-Bo Liu, ${ }^{1}$ and Menghao $\mathrm{Wu}^{1}$ \\ ${ }^{1}$ School of Physics and Wuhan National High Magnetic Field Center, Huazhong University of Science and Technology, Wuhan 430074, China \\ ${ }^{2}$ Institutes of Physical Science and Information Technology, Anhui University, Hefei, Anhui 230601, China
}

(Received 23 September 2020; revised 3 December 2020; accepted 7 December 2020; published 22 December 2020)

\begin{abstract}
Single-molecule magnets (SMMs) possessing bistable states have been considered as promising candidates to realize zero-dimensional (OD) ferroelectrics (FE) and multiferroics (MF) with high storage density. However, how to read or manipulate the FE states with low-energy-dissipation strategy is still a hard challenge. Here, we intercalated a magnetic metal porphyrin molecule, such as TiP with switchable vertical electric polarization, within the $\mathrm{MoS}_{2}$ bilayer to realize OD FE states. First-principles calculations show that the $\mathrm{MoS}_{2}$ monolayer contacted by SMMs is spin polarized to provide two spin-dependent transport tunnels. As a temperature gradient is applied along the transport channels, a well-defined spin-Seebeck effect (SSE) occurs in the spin-polarized $\mathrm{MoS}_{2}$ layer, helping to generate a pure thermal spin current. More importantly, the spin-Seebeck signal is associated tightly with the FE state in the same layer, and both of them can be switched simultaneously to another layer by an external electric field. The theoretical results not only put forwards a low-energy-dissipation strategy to read the SMM-based FE states, but also develop a new research field of spin-ferroelecto-caloritronics, which focuses on the interplay of electrons' spin and FE states in the presence of a temperature gradient.
\end{abstract}

DOI: 10.1103/PhysRevResearch.2.043406

\section{INTRODUCTION}

Ferroelectrics and multiferroics have long been attractive for researchers in materials science and condensed matter physics because they provide new physical mechanisms for realizing information storage and processing with low-energy dissipation [1-10]. To develop the ferroelectric (FE) materials towards realistic device applications, two challenging issues need to be addressed-one is how to increase the data-storage density with FE domains and the other is how to improve the flexibility of FE materials for the easy control [11-13]. One of the feasible ways is to reduce the dimensionality of FE materials [14]. On the other hand, single-molecule magnets (SMMs) are currently subject of active interdisciplinary of research, owning to their peculiar quantum spin states and slow magnetic relaxation, which are potentially suitable for quantum computing and sensing applications [15-17]. Recently, zero-dimensional (OD) multiferroic (MF) materials have been integrated with SMMs constructed on polar metal porphyrin (MP) molecules such as TiP, VP, and TiP, which have buckled structures with switchable vertical electric polarizations to realize two opposite FE states [18-22]. This design is useful to obtain $0 \mathrm{D}$ quantum materials, which are characterized by their peculiar couplings between spin states and FE states. To obtain stable and controllable OD FE states, we may

\footnotetext{
*Corresponding author: hhfu@ hust.edu.cn

Published by the American Physical Society under the terms of the Creative Commons Attribution 4.0 International license. Further distribution of this work must maintain attribution to the author(s) and the published article's title, journal citation, and DOI.
}

intercalate the SMMs within the bilayer structures constructed on two-dimensional (2D) materials such as $\mathrm{MoS}_{2}, \mathrm{MoSe}_{2}$, and $\mathrm{MoTe}_{2}$ [19]. One of the advantages of this design is that the FE states induced by OD multiferroics can be switched and manipulated easily by multiple feasible ways including an external electric field, and the other is that the information storing density can be enhanced remarkably, because every molecule in the SMMs can store even up to 1 bit data.

The material candidates to construct OD FE states are usually semiconducting or insulating, which brings us a challenge how to realize the readout and manipulation of FE states with low-energy-dissipation strategies. To achieve feasible routes to read and detect the FE states, here we focus on the geometric structures of the aforementioned SMM-based OD FE materials [19], and choose a SMM such as TiP molecule and a $2 \mathrm{D}$ material such as the $\mathrm{MoS}_{2}$ monolayer as examples to construct OD MF states. Here, TiP molecules, i.e., Ti porphyrin molecules, are widely present in many biochemical molecules, such as iron porphyrin in hemoglobin for oxygen transport in the blood. As illustrated in Figs. 1(a) and 1(b), a single TiP molecule is absorbed by a $\mathrm{MoS}_{2}$ monolayer, and Ti atom in the SMM is coupled with the S atom in the $\mathrm{MoS}_{2}$ monolayer. The single TiP molecule is a polar one characterized by two typical bistable states, in which the central Ti atom can shift upwards and downwards perpendicular to the molecule surface to realize two opposite electric polarizations. To ensure the stability of FE states, the TiP molecule is absorbed by the $\mathrm{MoS}_{2}$ monolayer to form the Ti-S bond with an effective coupling, as described in Fig. 1(b). The first-principles calculations show that the spin splitting occurs in the $\mathrm{MoS}_{2}$ monolayer due to the spin coupling with the TiP SMM. To illustrate this point, one can be refer to their spin distributions in every repeated unit drawn in Fig. 1(c), and the 
(a)

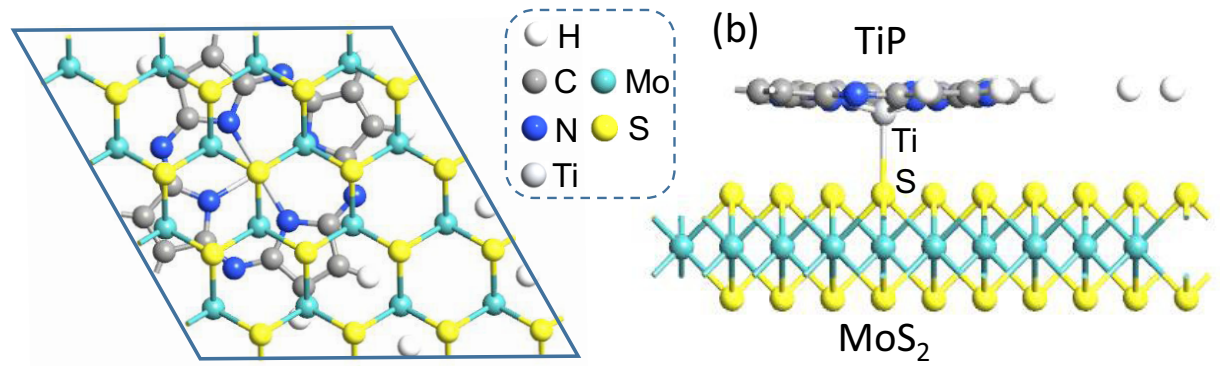

(c)

(d)

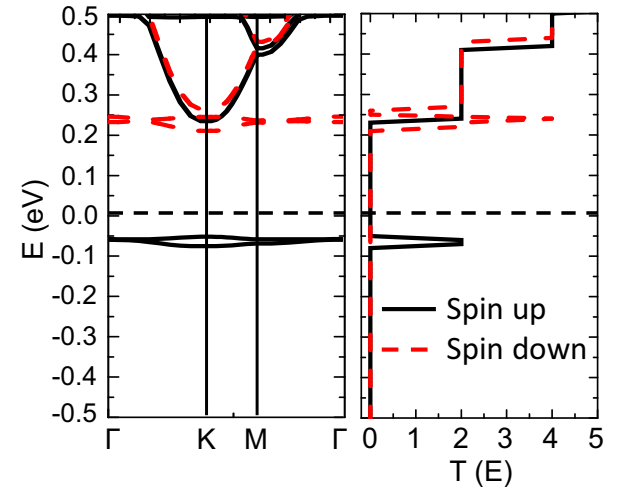

FIG. 1. (a, b) Top and side views of $\mathrm{MoS}_{2}$ monolayer adsorbed by a single magnetic TiP molecule, where Ti atom in the single molecule is coupled with the S atom in $\mathrm{MoS}_{2}$. (c) Spin distributions in the $\mathrm{MoS}_{2}$ monolayer and the TiP molecule. (d) Band structure of MoS 2 monolayer coupled with single TiP molecules and (e) spin-dependent transmission of the $\mathrm{MoS}_{2}$ monolayer coupled with a list of TiP molecules, in which the length of Ti-S bond and the distance between two neighboring SMMs are adopted as the same as those in (b).

spin polarized band structures in $\mathrm{MoS}_{2}$ monolayer drawn in Fig. 1(d). We find that the highest occupied molecular orbital (HOMO) and lowest unoccupied molecular orbital (LUMO) bands are constructed only by the spin-up and spin-down electrons, respectively, and divided by an energy gap about $0.3 \mathrm{eV}$. Moreover, these spin-splitting bands around the Fermi level are contributed to mainly by the $\mathrm{S}$ atoms in $\mathrm{MoS}_{2}$ monolayer, indicating that two spin transmission channels, one for the spin-up electrons and the other for the spin-down ones, appear in the $\mathrm{MoS}_{2}$ monolayer, as illustrated in Fig. 1(e).

In this work, we constructed spin caloritronic devices based on the $\mathrm{MoS}_{2}$ monolayer or bilayer intercalated by TiP SMMs. The theoretical calculations reveal that as a temperature gradient is applied in the $\mathrm{TiP}-\mathrm{MoS}_{2}$-bilayer device, and by adjusting the direction of an external electric field, the spin-Seebeck currents are driven in the different $\mathrm{MoS}_{2}$ layer, which is associated tightly with the different FE states in TiP SMMs, meaning that the spin-Seebeck signals connect with the related FE states in the TiP-MoS 2 -bilayer-based device. These properties not only indicate that we obtain a new lowenergy-dissipation strategy, i.e., by detecting the spin-Seebeck signal in the different $\mathrm{MoS}_{2}$ layer, to read the corresponding FE state in the SMM, but also develop a new interdisciplinary research field of spin-ferroelecto-caloritronics, which focuses on the interplay of electrons' spin and FE states in the presence of temperature gradient.

The reminder of this paper is organized as follows. The spin caloritronic devices based on the $\mathrm{MoS}_{2}$ monolayer or bilayer intercalated by TiP SMMs are designed, and the theoretical methods are introduced in details in Sec. II, then the numerical results for the thermally driven charge and spin currents to confirm the occurrence of the spin-Seebeck effect (SSE) are presented and how the spin-Seebeck signals are associated with the corresponding FE states is explained in Sec. III. In the last section, the main conclusions are summarized.

\section{DEVICE MODELS AND THEORETICAL METHOD}

Considering the unique spin-splitting band structures of TiP-coupled $\mathrm{MoS}_{2}$ monolayer, and the fact that occurrence of spin splitting in $\mathrm{MoS}_{2}$ monolayer is tightly associated with the FE state in the TiP SMM, we may explore new routes to read and manipulate these 0D FE states. To realize this idea, we try to design a spintronic device based on the above-mentioned TiP-coupled $\mathrm{MoS}_{2}$ monolayer, as drawn in Fig. 2(a), where three typical device regions, i.e., the left lead, the central scattering region, and the right lead, are constructed. To perform the structural optimization on the TiP-coupled-MoS ${ }_{2}$ monolayer-based devices, we first performed the theoretical calculations based on density functional theory (DFT) methods implemented in the Vienna $a b$ initio Simulation Package codes (VASP) [23,24]. The exchange-correlation effect is described within the generalized gradient approximation (GGA) in the Perdew-Burke-Ernzerhof (PBE) functional [25], together with the projector augmented wave method [26]. The Brillouin zone integration of the supercell in $2 \mathrm{D}$ materials is sampled with a $5 \times 5 \times 1$ Monkhorst-Pack [27] grid, the kinetic energy cutoff is set to be $400 \mathrm{eV}$, and a vacuum space of $40 \AA$ is set in the vertical direction. PBE-D2 functional of Grimme [28] is used to account for weak van der Waals interactions. For TiP-coupled-MoS 2 -monolayer-based device, 

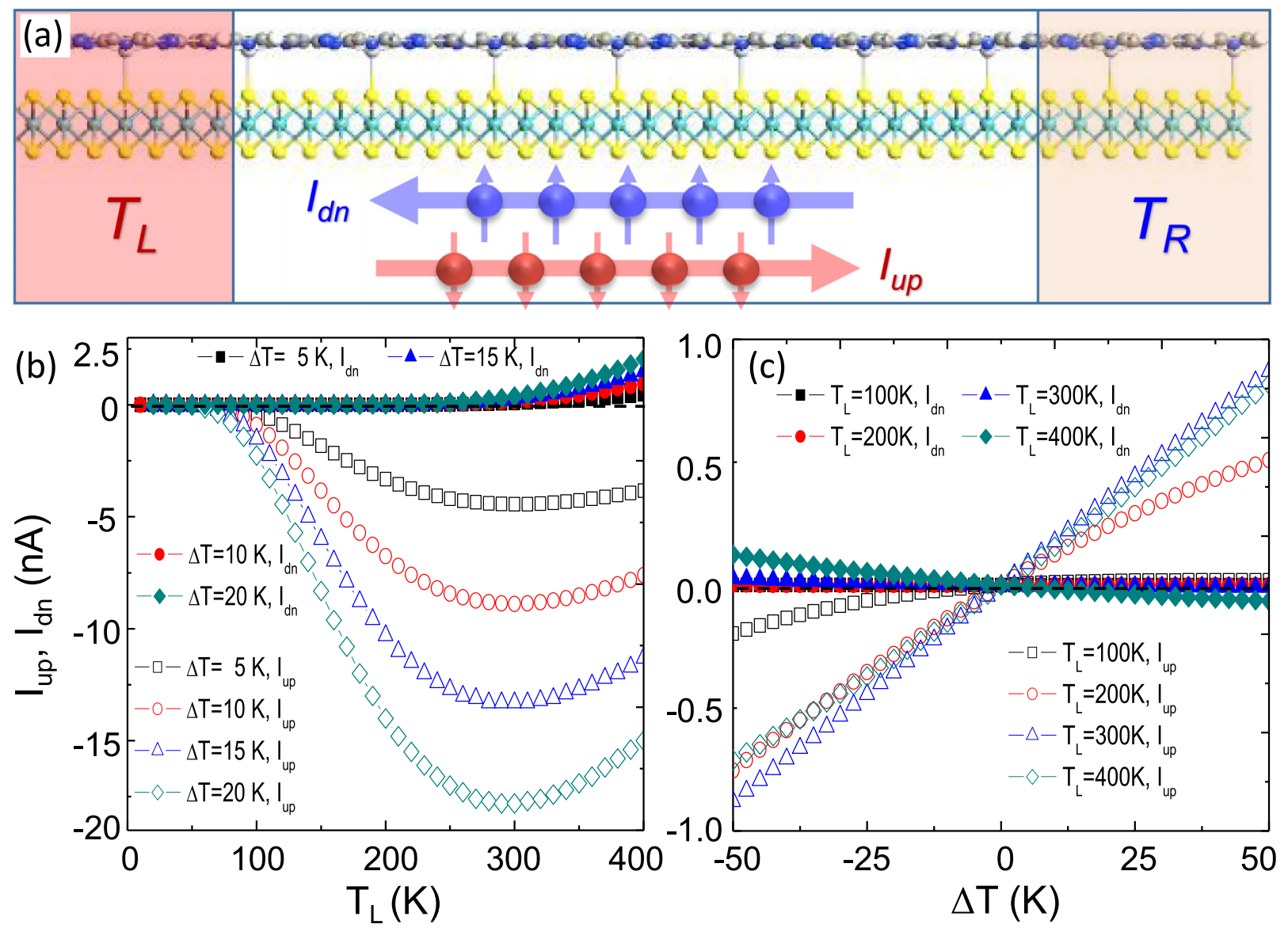

FIG. 2. (a) Schematic of the TiP-coupled-MoS $\mathrm{M}_{2}$-based spin-caloritronic device, where the semi-infinite left and right leads are composed of the same $\mathrm{MoS}_{2}$ monolayer with the temperature $T_{L}$ and $T_{R}$, respectively. The spin currents $I_{u p}$ and $I_{d n}$ and their flow directions are denoted by the arrows with different colors. (b) The thermally driven spin currents $I_{u p}$ and $I_{d n}$ versus the temperature $T_{L}$ at the different temperature gradient $\Delta T$. (c) The spin-dependent currents $I_{u p}$ and $I_{d n}$ versus $\Delta T$ at the different temperature $T_{L}$.

to eliminate the interaction between adjacent molecules, a $2 \times 2$ supercell of $\mathrm{MoS}_{2}$ monolayer is adopted so that the distances between the hydrogen atoms on the edges of two adjacent molecules are greater than $3.7 \AA$, and thus the interaction between them might be negligible. Furthermore, to derive the electrons to transport in the $\mathrm{MoS}_{2}$ monolayer, the temperatures in the left and right leads are set as $T_{L}$ and $T_{R}$, respectively, and a temperature gradient $\Delta T\left(=T_{L}-T_{R}\right)$ is adopted. Owing to two spin-dependent transmission channels distributed nearly symmetrically above and below the Fermi level, two spin-dependent currents, i.e., the spin-up current $I_{u p}$ and the spin-down one $I_{d n}$, may be driven by the temperature gradient to flow in the opposite directions, as displayed in Fig. 2(a). The appearance of thermally driven spin-dependent currents characterized by the above two typical features indicates that the conduction electron-based SSE [29-36] occurs in the present spintronic device based on the TiP-coupled $\mathrm{MoS}_{2}$ monolayer.

To confirm the occurrence of SSE in the present TiPabsorbed-MoS $\mathrm{S}_{2}$-based spintronic device, we may numerically calculate the thermally driven spin current $I_{u p}$ and $I_{d n}$ by using the DFT $[37,38]$ combined with the nonequilibrium Green's function (NEGF) approach [39-42], as implemented in the SIESTA and the TRANSAMPA codes [43]. In the LandauerBüttiker formalism [44], the spin-dependent currents are given by [45]

$$
I_{\sigma}=\frac{e}{h} \int_{-\infty}^{+\infty}\left\{T_{\sigma}(E)\left[f_{L}\left(E, T_{L}\right)-f_{R}\left(E, T_{R}\right)\right]\right\} d E,
$$

where $e$ is the electronic charge, $h$ is the Plank constant, and the equilibrium Ferpmi-Dirac distribution of the left (right) lead at temperature $T_{L(R)}$ is given by the equation $f_{L(R)}\left(E, T_{L(R)}\right)=\left[1+\exp \left(E-\mu_{L(R)}\right) / k_{B} T_{L(R)}\right]^{-1} \cdot \mu_{L(R)}$ is the chemical potential in the left (right) lead. $T_{\sigma}(E)$ is the spin-resolved transmittance function, which is defined as $T_{\sigma}(E)=\operatorname{Tr}\left[\Gamma_{L}^{\sigma} G_{\sigma}^{R}(E) \Gamma_{R}^{\sigma} G_{\sigma}^{A}(E)\right][25]$, where $\sigma(=\uparrow, \downarrow$ or $u p, d n$ ) denotes the spin index, and $\Gamma_{L / R}^{\sigma}=i\left|\Sigma_{L / R}^{\sigma}-\Sigma_{L / R}^{\sigma}\right|$ indicates the interaction between a central scattering region and the left (right) lead, whose self-energy is $\Sigma_{L / R}^{\sigma}$. $G_{\sigma}^{R / A}(E)$ represents the retarded (advanced) Green's function of the central scattering region, $G_{\sigma}^{R}(E)=\left[H_{\sigma}^{c}-(E+\right.$ $\left.i \eta)+\Sigma_{L}^{\sigma}+\Sigma_{R}^{\sigma}\right]^{-1}$ and $G_{\sigma}^{A}(E)=\left[G_{\sigma}^{R}(E)\right]^{\dagger}$, here $H_{\sigma}^{c}$ is the Hamiltonian in the central scattering region. Considering that the SMM-coupled $\mathrm{MoS}_{2}$ monolayer designed here are structurally perfect, thermally driven electrons can move freely in the $\mathrm{MoS}_{2}$ monolayer. The above expressions will help us to obtain the thermal spin-dependent currents transport through the TiP-coupled $\mathrm{MoS}_{2}$ monolayer. 
In addition, to examine further the features of SSE, we also calculate the spin-dependent Seebeck coefficients, which measure the spin voltage induced by temperature gradient in the TiP-coupled-MoS $\mathrm{S}_{2}$-based devices. In the linear response regime, if $\Delta T$ approaches zero, we get $T=T_{L} \approx T_{R}$ and $\mu=\mu_{L} \approx \mu_{R}$ in the spin caloritronic device constructed here. Thus, the spin-dependent Seebeck coefficients $\left(S_{\sigma}\right)$ can be calculated by [46]

$$
S_{\sigma}(\mu, T)=-\frac{1}{e T} \frac{K_{\sigma, 1}(\mu, T)}{K_{\sigma, 0}(\mu, T)},
$$

where, $K_{\sigma, n}(\mu, T)$ is given by

$$
K_{\sigma, n}(\mu, T)=-\int_{-\infty}^{+\infty}(E-\mu)^{n} \frac{\partial f(E, \mu, T)}{\partial E} T_{\sigma}(E) d E .
$$

$S_{c h}=\left(S_{u p}+S_{d n}\right) / 2$ and $S_{s p}=S_{u p}-S_{d n}$ are the chargeSeebeck and spin-Seebeck coefficient. Note that in the theoretical calculations, the electron-phonon interaction (EPI) is ignored here since the electron-phonon mean free paths in the $\mathrm{MoS}_{2}$ monolayer are short to about $9 \mathrm{~nm}$ at room temperature as demonstrated in a previous literature [47], indicating that much weak EPI exists in the $\mathrm{MoS}_{2}$-monolayer-based devices.

\section{RESULTS AND DISCUSSION}

Now, we turn to elucidate how to readout the two opposite FE states by the related spin-Seebeck signals in TiP-coupled$\mathrm{MoS}_{2}$-based devices. When a temperature gradient $\Delta T$ is applied between two leads, $f_{L}\left(T_{L}\right)-f_{R}\left(T_{L}-\Delta T\right)$ is no longer equal to zero and displays inverse symmetric behaviors around the Fermi level [48,49]. Since $f_{L}-f_{R}$ is an odd function, the sign of $I_{\sigma}$ is determined by the slope of the transmission coefficient $T_{\sigma}$ near to the Fermi level, according to the aforementioned expressions of spin-dependent currents [34]. Considering that the spin-up and spin-down transmission spectra are located below and above the Fermi level, respectively, $I_{u p}$ and $I_{d n}$ should have the opposite signs, indicating the occurrence of conduction electron-based SSE in the device [50]. This property is confirmed further by the their numerical results as illustrated in Figs. 2(b) and 2(c), where $I_{u p}$ and $I_{d n}$ versus $T_{L}$ and $\Delta T$ are plotted, respectively. Indeed, one can see that $I_{u p}<0$ while $I_{d n}>0$ from the DFT calculations combined with the NEGF method. Moreover, $I_{u p}$ and $I_{d n}$ possess different threshold temperatures due to their different spin-dependent band gaps. This is to say that, in the low-temperature region of $T_{L}$, both $I_{u p}$ and $I_{d n}$ are forbidden, indicating that there is no any carrier transport to denote the related FE state. In the intermediate-temperature region, only $I_{u p}$ appears, indicating that the carrier transport is dominated by the absolute spin-polarized currents, and only as $T_{L}$ increases to larger values, the SSE occurs, which is helpful to reduce the charge current $I_{c}\left(=I_{u p}+I_{d n}\right)$ while to enhance the net spin current $I_{s}\left[=\frac{h}{2 e}\left(I_{u p}-I_{d n}\right)\right]$.

Furthermore, to confirm further the occurrence of SSE in the device, we also calculated the spin-dependent Seebeck coefficients $S_{u p}, S_{d n}, S_{c h}$, and $S_{s p}$ versus the device temperature $T$ and the potential $\mu$ in the TiP-coupled-MoS $\mathrm{S}_{2}$-based devices in Fig. S1 in the Supplemental Material [51]. One can find that changing trends of these coefficients versus the device temperature $T$ are much similar with those of $I_{u p}, I_{d n}, I_{c h}$, and $I_{s p}$ versus $T_{L}$. In particular, two spin-dependent Seebeck coefficients $S_{u p}$ and $S_{d n}$ possess the opposite signs, for all device temperature settings [Fig. S1(a)] or around the Fermi level (Fig. S1(b)], which is a typical characteristic of conduction electron-based SSE. Besides, the spin-Seebeck coefficients at low temperatures are high to $10^{4} \mu \mathrm{V} / \mathrm{K}$, supporting that the TiP-coupled $\mathrm{MoS}_{2}$ monolayer has the potential to work as good thermoelectric-conversion devices.

To obtain a low-energy-dissipation strategy to realize the readout of FE states in the $0 \mathrm{D}$ MF materials by using the spin-Seebeck signals, two key issues should be addressed: (i) how to combine the thermally driven spin currents with the corresponding FE states and (ii) how to reduce the thermal charge current $I_{c}$ and to enhance the spin current $I_{s}$ since the charge current usually brings Joule heat, which is not beneficial to design low-energy-dissipation nanodevices. To construct two FE states with opposite electric polarizations, we intercalate the SMM within a bilayer of 2D materials, such as the $\mathrm{MoS}_{2}$ bilayer adopted here. As illustrated in Figs. 3(a) and 3(b), the single TiP molecules are sandwiched between two $\mathrm{MoS}_{2}$ monolayers. Since the TiP molecule is polar and its electric polarization appears along the vertical direction of the molecule surface, we can apply an external electric field $\vec{E}$ to flip their polarization directions. If $\vec{E}$ is applied in the upwards direction, the TiP molecules are absorbed by the upper $\mathrm{MoS}_{2}$ monolayer, otherwise, the TiP molecules are absorbed by the lower monolayer. Thus, two opposite electric polarization states corresponding to two opposite FE states characterized by " 0 " and " 1 " are achieved. It should be pointed out that the previous first-principles calculations reveal that the potential barrier between these two opposite FE states is high to $0.73 \mathrm{eV}$ [19], which ensures the thermal stability of these electric-polarized states even at room temperature.

On the other side, as the magnetic TiP molecules are absorbed by the upper $\mathrm{MoS}_{2}$ monolayer, the later would be spin polarized to provide two spin-dependent transport channels as interpreted above, while the lower $\mathrm{MoS}_{2}$ monolayer keeps insulating [see Figs. 3(c) and 3(d)]. Thus, we can construct the spin-caloritronic device based on the TiP-intercalated $\mathrm{MoS}_{2}$ bilayers by using the similar way, as drawn in Figs. 3(e) and 3(f). In the present device geometrical structures, we can use the thermally driven spin currents to read or manipulate the corresponding FE state, thanking to the occurrence of the layer-dependent SSE in the system.

As for the second key issue, it is fortunate that we also obtain an effective way to reduce the thermal charge current, and even to get a nearly pure spin current without any charge accumulation to read the corresponding FE states. The $a b$ initio calculations show that the spin-splitting band structures of TiP-intercalated $\mathrm{MoS}_{2}$ bilayer can be adjusted effectively by controlling the Ti-S bond length $L_{\mathrm{Ti}-\mathrm{S}}$. It should be noted that as $L_{\mathrm{Ti}-\mathrm{S}}$ is adjusted, the spin distribution in the related $\mathrm{MoS}_{2}$ monolayer will be changed. However, from the previous first-principles calculations [19], the vertical distance between $\mathrm{Ti}$ and porphyrin plane is long to $0.42 \AA$, and the binding energy of $\mathrm{Ti}$ in the TiP molecule is high to $12 \mathrm{eV}$ per atom, much larger than the bulk cohesive energy of $\mathrm{Ti}$, revealing that the system of TiP-intercalated $\mathrm{MoS}_{2}$ bilayer is stable, and the electric-polarized state in TiP molecule is 

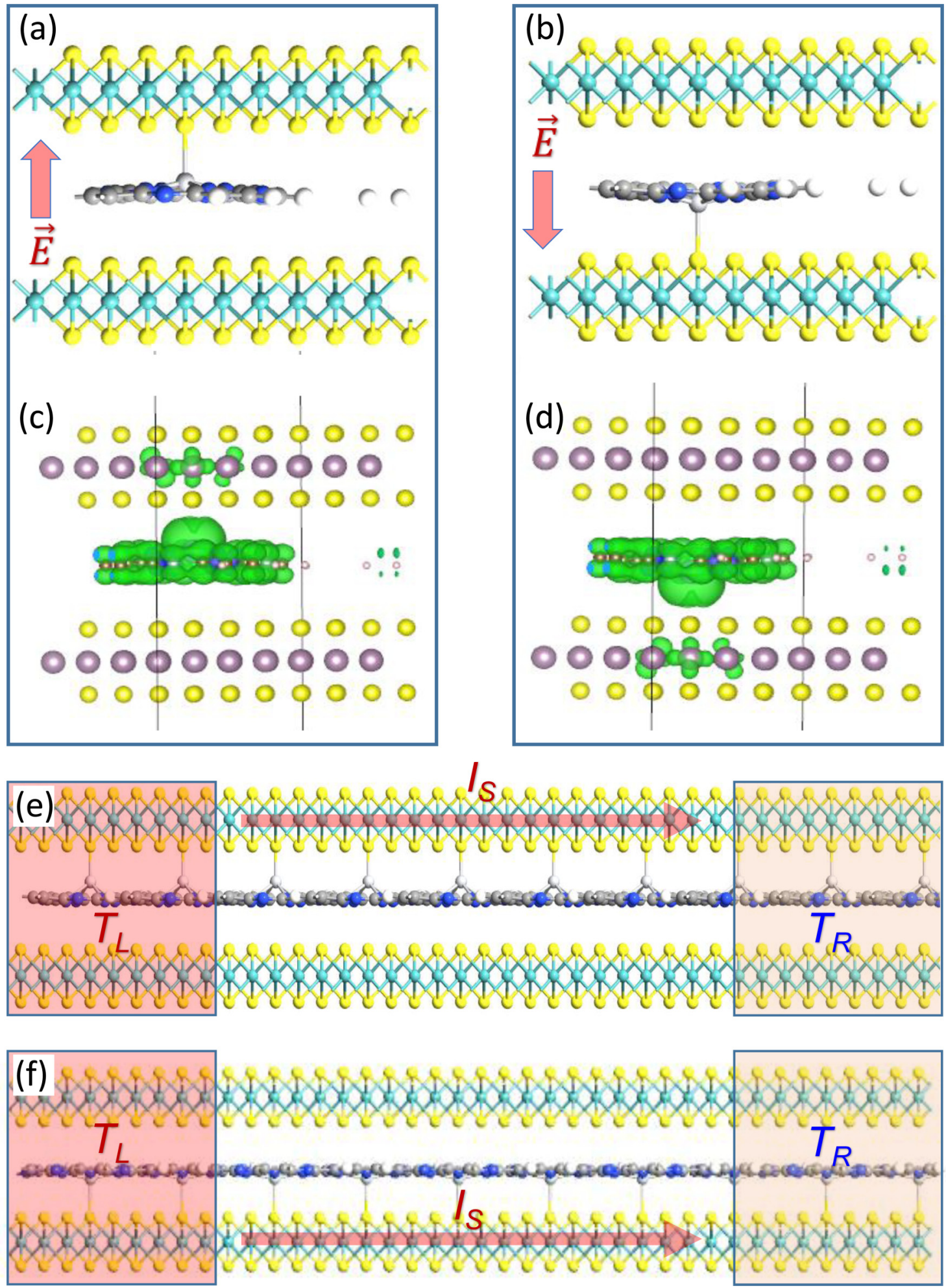

FIG. 3. (a, b) Two schematic structures of TiP-intercalated $\mathrm{MoS}_{2}$ bilayer, where the TiP molecule is sandwiched between two MoS 2 monolayers to produce two opposite FE states by flipping an external electric field. (c, d) The spin distributions of the related FE states. (e, f) Two schematics of the TiP-intercalated-MoS ${ }_{2}$-bilayer-based devices with the temperatures $T_{L}$ and $T_{R}$ to illustrate the occurrence of layer-dependent SSE.

less influenced by the coupling between $\mathrm{Ti}$ and $\mathrm{S}$ atoms. In Figs. 4(a) to 4(d), we plotted the band structures of the TiP$\mathrm{MoS}_{2}$ bilayer as $L_{\mathrm{Ti}-\mathrm{S}}$ is increased from 13.9967 to $13.5967 \AA$ by a step of $0.2 \AA$. One can find that with the increasing of
$L_{\mathrm{Ti}-\mathrm{S}}$, the spin-splitting energy bands shift downwards, making the spin-up and spin-down bands tend to be symmetrical about the Fermi level, indicating that two nearly symmetrical spin-dependent transport channels form in the spin-splitting 

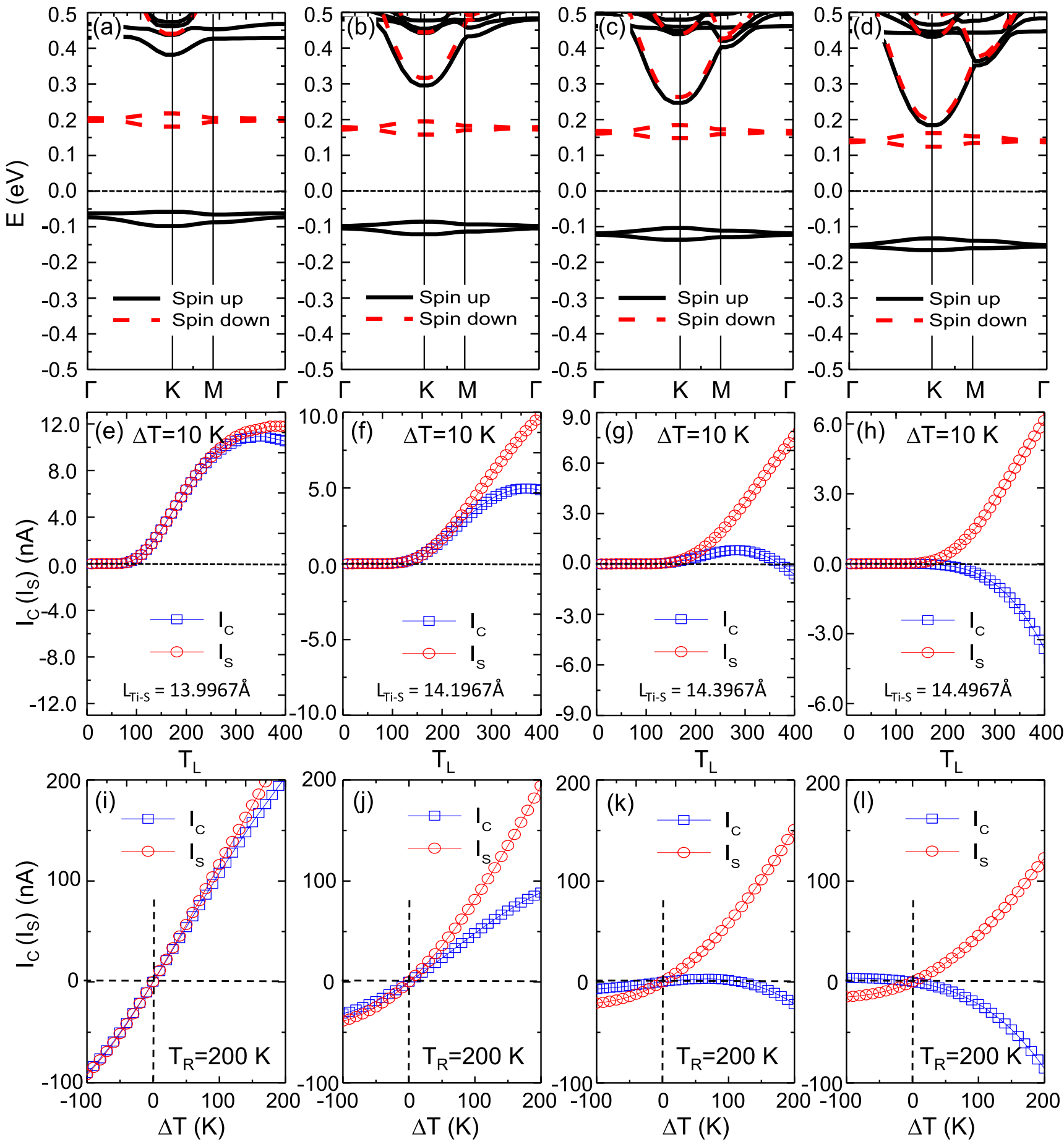

FIG. 4. (a)-(d) Spin-dependent band structures of TiP-intercalated bilayer $\mathrm{MoS}_{2}$ when the length of Ti-S bond $L_{\mathrm{Ti}-\mathrm{S}}$ gradually changes from 13.9967 to $14.5967 \AA$. (e)-(h) The charge current $I_{c}$ and the spin current $I_{s}$ of the related spin caloritronic devices versus the temperature $T_{L}$ in the left lead at the temperature gradient $\Delta T=10 \mathrm{~K}$. (i)-(l) $I_{c}$ and $I_{s}$ versus $\Delta T$ at the device temperature $T_{R}=200 \mathrm{~K}$.

transmission spectra. The numerical results for the thermally driven charge current $I_{c}$ and the spin current $I_{s}$ support the above conclusion. In Figs. 4(e) and 4(f), we plotted $I_{c}$ and $I_{s}$ versus $T_{L}$ for $\Delta T=10 \mathrm{~K}$, and in Figs. 4(i) to 4(1), plotted $I_{c}$ and $I_{s}$ versus $\Delta T$ at $T_{R}=200 \mathrm{~K}$ for the TiP-intercalated$\mathrm{MoS}_{2}$-bilayer-based devices. It is interesting that as $L_{\mathrm{Ti}-\mathrm{S}}$ increases, $I_{c}$ is reduced remarkably as $T_{L}$ or $\Delta T$ increases, while $I_{s}$ keeps finite values for any case. In particular, for the device with $L_{\mathrm{Ti}-\mathrm{S}}=14.3967 \AA, I_{c}$ is reduced nearly to zero in a large region of device temperature settings, indicating that a nearly pure spin current is obtained in the device. Note that the corresponding spin-Seebeck coefficients adjusted by the strength of Ti-S bonds confirm further the above findings (see Figs. S2 and S3 in the Supplemental Material [51]). These properties indicate that we obtain an effective way to read the corresponding FE states without any energy dissipation since the charge current is forbidden while the nearly pure spin current is generated. Moreover, by using the inverse spin Hall 
effect (ISHE) [52,53], the pure spin current can be converted to an observable voltage between two boundaries of the $\mathrm{MoS}_{2}$ monolayer, which can be detected directly in experiments.

It should be noted that the physical mechanism of spin-Seebeck signals in the present TiP-intercalated-MoS ${ }_{2}$ bilayer-based device can be expanded to other OD FE systems, which are composed of VP molecules intercalated between $\mathrm{MoSe}_{2}$ or MoTe 2 bilayer (see Figs. S4 to S7 in the Supplemental Material [51], for more details). Thus, we believe that the response signals from the SSE provide us an effective route to realize the readout and manipulation of the FE states in OD MF materials.

\section{CONCLUSION}

In summary, we designed theoretically some spin caloritronic devices based on OD MF materials, which are constructed by the SMMs, such as the magnetic TiP molecules, intercalated within 2D materials, such as the $\mathrm{MoS}_{2}$ bilayer. The SMMs can exhibit two opposite FE states as they are absorbed by the upper or lower $\mathrm{MoS}_{2}$ monolayer, which can

[1] K. Chang, J. Liu, H. Lin, N. Wang, K. Zhao, A. Zhang, F. Jin, Y. Zhong, X. Hu, W. Duan, Q. Zhang, L. Fu, Q.-K. Xue, X. Chen, and S.-H. Ji, Discovery of robust in-plane ferroelectricity in atomic-thick SnTe, Science 353, 274 (2016).

[2] J. Long, M. S. Ivanov, V. A. Khomchenko, E. Mamontova, J. M. Thibaud, J. Rouquette, M. Beaudhuin, D. Granier, R. A. S. Ferreira, L. D. Carlos, B. Donnadieu, M. S. C. Henriques, J. A. Paixao, Y. Guari, and J. Larionova, Room temperature magnetoelectric coupling in a molecular ferroelectric ytterbium(III) complex, Science 367, 671 (2020).

[3] S. E. Rowley and G. G. Lonzarich, Ferroelectrics in a twist, Nat. Phys. 10, 907 (2014).

[4] L. Li, Y. Yu, G. J. Ye, Q. Ge, X. Qu, H. Wu, D. Feng, X. H. Chen, and Y. Zhang, Black phosphorus field-effect transistors, Nat. Nanotechnol. 9, 372 (2014).

[5] S. N. Shirodkar and U. V. Waghmare, Emergence of Ferroelectricity at a Metal-Semiconductor Transition in a $1 \mathrm{~T}$ Monolayer of $\mathrm{MoS}_{2}$, Phys. Rev. Lett. 112, 157601 (2014).

[6] C. Huang, Y. Du, H. Wu, H. Xiang, K. Deng, and E. Kan, Prediction of Intrinsic Ferromagnetic Ferroelectricity in a Transition-Metal Halide Monolayer, Phys. Rev. Lett. 120, 147601 (2018).

[7] C. Xiao, F. Wang, S. A. Yang, Y. Lu, Y. Feng, and S. Zhang, Elemental ferroelectricity and antiferroelectricity in group-V monolayer, Adv. Funct. Mater. 28, 1707383 (2018).

[8] M. Wu, S. Dong, K. Yao, J. Liu, and X. C. Zeng, Ferroelectricity in covalently functionalized two-dimensional materials: integration of high-mobility semiconductors and nonvolatile memory, Nano. Lett. 16, 7309 (2016).

[9] Y. Zhao, L. Lin, Q. Zhou, Y. Li, S. Yuan, Q. Chen, S. Dong, and J. Wang, Surface vacancy-induced switchable electric polarization and enhanced ferromagnetism in monolayer metal trihalides, Nano. Lett. 18, 2943 (2018).

[10] W. Mu and X. C. Zeng, Bismuth oxychalcogenides: a new class of ferroelectric/ferroelastic materials with ultra high mobility, Nano. Lett. 17, 6309 (2017). be switched by an external electric field. The $a b$ initio calculations combined with the NEGF theory show that the $\mathrm{MoS}_{2}$ monolayer coupled with the SMMs are spin polarized to provide two spin channels for spin-up and spin-down electrons, respectively. As a temperature gradient is applied in the above $\mathrm{MoS}_{2}$ monolayer, a well-defined conduction electron-based SSE occurs, and the spin current generated in the $\mathrm{MoS}_{2}$ monolayer is tightly combined with the FE state in the coupled TiP molecule. By adjusting the length of Ti-S bonds, a pure thermal spin current can be achieved. These transport characteristics support that we can apply the spin-Seebeck signals to realize the readout of the FE states in OD FE materials. Our theoretical results not only put forwards a low-powerdissipation strategy to read the SMM-based FE states, but also develop a new research field of spin-ferroelecto-caloritronics, focusing on the interplay of electrons' spin and the FE states in the presence of temperature gradient.

\section{ACKNOWLEDGMENTS}

This work is supported by the National Natural Science Foundation of China with Grant No. 11774104.

[11] M. Dawber, K. M. Rabe, and J. F. Scott, Physics of thin-film ferroelectric oxides, Rev. Mod. Phys. 77, 1083 (2005).

[12] T. Kimura, T. Goto, H. Shintani, K. Ishizaka, T. Arima, and Y. Tokuram, Magnetic control of ferroelectric polarization, Nature 426, 55 (2003).

[13] J. F. Scott, Applications of modern ferroelectrics, Science 315, 954 (2007).

[14] A. V. Bune, V. M. Fridkin, S. Ducharme, L. M. Blinov, S. P. Palto, A. V. Sorokin, S. G. Yudin, and A. Zlatkin, Twodimensional ferroelectric films, Nature 391, 874 (1998).

[15] R. Sessoli, D. Gatteschi, A. Caneschi, and M. A. Novak, Magnetic bistability in a metal-ion cluster, Nature 365, 141 (1993).

[16] M. N. Leuenberger and D. Loss, Quantum computing in molecular magnets, Nature 410, 789 (2001).

[17] M. Yamanouchi, D. Chiba, F. Matsukura, and H. Ohno, Current-induced domain-wall switching in a ferromagnetic semiconductor structure, Nature 428, 539 (2004).

[18] S. Horiuchi, Y. Tokunaga, G. Giovannetti, S. Picozzi, H. Itoh, R. Shimano, R. Kumai, and Y. Tokura, Ahove-room-temperature ferroelectricity in a single-component molecular crystal, Nature 463, 789 (2010).

[19] Q. Yang, T. T. Zhong, Z. Tu, L. Zhu, M. Wu, and X. C. Zeng, Design of single-molecule multiferroics for efficient ultrahighdensity nonvolatile memories, Adv. Sci. 6, 1801572 (2019).

[20] P. Liljeroth, J. Repp, and G. Meyer, Current-induced hydrogen tautomerization and conductance switching of naphthalocyanine molecules, Science 317, 1203 (2007).

[21] T. Huang, J. Zhao, M. Feng, A. A. Popov, S. Yang, L. Dunsch, and H. Petek, A molecular switch based on current-driven rotation of an encapsulated cluster within a fullerene cage, Nano Lett. 11, 5327 (2011).

[22] P. H. Guo, Y. Meng, Y. C. Chen, Q. W. Li, B. Y. Wang, J. D. Leng, D. H. Bao, J. H. Jia, and M. L. Tong, A zigzag Dy4(III) cluster exhibiting single-molecule magnet, ferroelectric and white-light emitting properties, J. Mater. Chem. C 2, 8858 (2014). 
[23] G. Kresse and J. Furthmüller, Efficiency of ab-initio total energy calculations for metals and semiconductors using a plane-wave basis set, Comput. Mater. Sci. 6, 15 (1996).

[24] G. Kresse and J. Furthmüller, Efficient iterative schemes for ab initio total-energy calculations using a plane-wave basis set, Phys. Rev. B 54, 11169 (1996).

[25] J. P. Perdew, K. Burke, and M. Ernzerhof, Generalized Gradient Approximation Made Simple, Phys. Rev. Lett. 77, 3865 (1996).

[26] P. E. Blöchl, Projector augmented-wave method, Phys. Rev. B 50, 17953 (1994).

[27] H. J. Monkhorst and J. D. Pack, Special points for Brillouin-zone integrations, Phys. Rev. B 13, 5188 (1976).

[28] S. Grimme, Semiempirical GGC-type density functional constructed with a long-range dispersion contribution, J. Comput. Chem. 27, 1787 (2006)

[29] G. E. Bauer, E. Saitoh, and B. J. van Wees, Spin caloritronics, Nat. Mater. 11, 391 (2012).

[30] X. Shi and L. Chen, Thermoelectric materials step up, Nat. Mater. 15, 691 (2016).

[31] K. Uchida, S. Takahashi, K. Harii, J. Ieda, W. Koshibae, K. Ando, S. Maekawa, and E. Saitoh, Observation of the spin Seebeck effect, Nature 455, 778 (2018).

[32] C. M. Jaworski, J. Yang, S. Mack, D. D. Awschalom, J. P. Heremans, and R. C. Myers, Observation of the spin-Seebeck effect in a ferromagnetic semiconductor, Nat. Mater. 9, 898 (2010).

[33] J. Xiao, G. E. W. Bauer, K. Uchida, E. Saitoh, and S. Maekawa, Theory of magnon-driven spin Seebeck effect, Phys. Rev. B 81, 214418 (2010).

[34] D.-D. Wu, H.-H. Fu, Q.-B. Liu, G.-F. Du, and R. Wu, Magnetic nanotubes: a new material platform to realize a robust spinSeebeck effect and a perfect thermal spin-filtering effect, Phys. Rev. B 98, 115422 (2018).

[35] H.-H. Fu, G.-F. Du, D.-D. Wu, Q.-B. Liu, and R. Wu, Spin-orbit coupling induced robust spin-Seebeck effect and pure thermal spin currents in achiral molecule systems, Phys. Rev. B 100, 085407 (2019).

[36] H.-H. Fu, D.-D. Wu, L. Gu, M.-H. Wu, and R. Wu, Design for a spin-Seebeck diode based on two-dimensional materials, Phys. Rev. B 92, 045418 (2015).

[37] M. Brandbyge, J. L. Mozos, P. Ordejòn, J. Taylor, and K. Stokbro, Density-functional method for nonequilibrium electron transport, Phys. Rev. B 65, 165401 (2002).

[38] J. E. Padilha, M. P. Lima, A. J. R. da Silva, and A. Fazzio, Bilayer graphene dual-gate nanodevice: An ab initio simulation, Phys. Rev. B 84, 113412 (2011).
[39] H.-H. Fu and K.-L. Yao, Perfect thermal spin filter and pure spin thermoelectric generator based on a laterally coupled double quantum-dot array, Europhys. Lett. 103, 57011 (2013).

[40] H.-H. Fu, L. Gu, D.-D. Wu, and Z.-Q. Zhang, Enhancement of the thermoelectric figure of merit in DNA-like systems induced by Fano and Dicke effects, Phys. Chem. Chem. Phys. 17, 11077 (2015).

[41] H.-H. Fu and K.-L. Yao, Influence of interdot hopping and intradot many-body interaction on conductance through parallel triple-quantum-dot device: Nonequilibrium Green's function approach, J. Appl. Phys. 108, 084510 (2010).

[42] H.-H. Fu and K.-L. Yao, Perfect spin-filter and quantum-signal generator in a parallel coupled multiple triple-quantum-dots device, J. Appl. Phys. 111, 124510 (2012).

[43] J. Taylor, H. Guo, and J. Wang, Ab initio modeling of quantum transport properties of molecular electronic devices, Phys. Rev. B 63, 245407 (2001).

[44] Y. Imry and R. Landauer, Conductance viewed as transmission, Rev. Mod. Phys. 71, S306 (1999).

[45] S. Datta, Electronic Transport in Mesoscopic Systems (Cambridge University Press, Cambridge, England, 1995).

[46] L. D. Hicks and M. S. Dresselhaus, Thermoelectric figure of merit of a one-dimensional conductor, Phys. Rev. B 47, 16631(R) (1993)

[47] W. Li, Electrical transport limited by electron-phonon coupling from Boltzmann transport equation: An ab initio study of $\mathrm{Si}, \mathrm{Al}$, and $\mathrm{MoS}_{2}$, Phys. Rev. B 92, 075405 (2015).

[48] M. Zeng, Y. Feng, and G. Liang, Graphene-based Spin caloritronics, Nano. Lett. 11, 1369 (2011)

[49] D.-D. Wu, Q.-B. Liu, H.-H. Fu, and R. Wu, How to realize a spin-dependent Seebeck diode effet in metallic zigzag gammagraphyen nanoribbons? Nanoscale 9, 18334 (2017).

[50] S. R. Boona, R. C. Myers, and J. P. Heremans, Spin caloritronics, Energ. Envir. Sci. 7, 885 (2014).

[51] See Supplemental Material at http://link.aps.org/supplemental/ 10.1103/PhysRevResearch.2.043406 for spin-Seebeck coefficients of the TiP-coupled- $\mathrm{MoS}_{2}$-monolayer-based device; spin-dependent band structures of the TiP- and VP-intercalated bilayer $\mathrm{MoS}_{3}, \mathrm{MoSe}_{2}$, and $\mathrm{MoTe}_{2}$; thermally driven spindependent currents; and the corresponding spin-Seebeck coefficients in the related devices constructed on the above SMMs and 2D materials.

[52] E. Saitoh, M. Ueda, H. Miyajima, and G. Tatara, Conversion of spin current into charge current at room temperature: Inverse spin-Hall effect, Appl. Phys. Lett. 88, 182509 (2006).

[53] K. Ando, Y. Kajiwara, S. Takahashi, S. Maekawa, K. Takemoto, M. Takatsu, and E. Saitoh, Conversion of spin current into charge current at room temperature: Inverse spin-Hall effect, Phys. Rev. B 78, 014413 (2008). 